\title{
Germanica
}

\section{Anne D. Peiter, Träume der Gewalt. Studien der Unverhältnismäßigkeit zu Texten, Filmen und Fotografien. Nationalsozialismus - Kolonialismus - Kalter Krieg}

Katja Schubert

\section{OpenEdition Journals}

Édition électronique

URL : https://journals.openedition.org/germanica/9223

DOI : 10.4000/germanica.9223

ISSN : 2107-0784

\section{Éditeur}

Université de Lille

\section{Édition imprimée}

Date de publication : 1 juin 2020

Pagination : 201-204

ISBN : 978-2-913857-45-2

ISSN : 0984-2632

\section{Référence électronique}

Katja Schubert, „Anne D. Peiter, Träume der Gewalt. Studien der Unverhältnismäßigkeit zu Texten, Filmen und Fotografien. Nationalsozialismus - Kolonialismus - Kalter Krieg", Germanica [Online], 66। 2ème trimestre 2020, Online erschienen am: 01 Januar 2021, abgerufen am 08 Januar 2022. URL: http://journals.openedition.org/germanica/9223 ; DOI: https://doi.org/10.4000/germanica.9223 


\title{
Anne D. Peiter, Träume der Gewalt. Studien der Unverhältnismäßigkeit zu Texten, Filmen und Fotografien. Nationalsozialismus - Kolonialismus - Kalter Krieg
}

\author{
Katja Schubert
}

\section{RÉFÉRENCE}

Bielefeld, Transkript, 2019, $1104 \mathrm{p}$.

1 „Eine Zumutung!“, könnte man ausrufen, wenn man das Buch von etwas mehr als tausend Seiten, die Publikation der Habilitation von Anne D. Peiter, Dozentin für Literatur- und Kulturwissenschaften im Fachbereich Germanistik an der Université de l'Ile de la Réunion, in die Hand nimmt. Tausend Seiten, wer kann so etwas lesen als wissenschaftliche Arbeit? Wer hat die Zeit und Ruhe zu solcher monströsen Lektüre, wie ist das zu schaffen in unserer schnelllebigen Epoche der Massenproduktion auch von wissenschaftlichen Publikationen? Vorab sei bemerkt, dass man das in drei Teile gegliederte Buch auch als drei unabhängige Bände lesen könnte, wenn man sich auf einen der Zeiträume beschränken möchte.

2 Tatsächlich sind wir aber mit dem Ausruf der Zumutung gar nicht so weit entfernt von dem, was vielleicht als so etwas wie ein Kern der Arbeitsweise und der Inhalte dieser umfassenden, weit ausgreifenden Arbeit bezeichnet werden kann. Denn die Frage nach Kontinuitäten und Brüchen in der Geschichte der modernen Gewalt zwischen dem 19. und dem 21. Jahrhundert, ausgehend vom Nationalsozialismus, zurückverweisend auf den Kolonialismus und vorausblickend auf die atomare Zerstörung kann gar nichts anderes sein als eine große Zumutung, die bei Lesenden und Forschenden Schwindel 
erzeugt. Alles ist unverhältnismäßig darin, so die Autorin, wenn man beginnt, sich auf das Über- und Unmaß von Gewalt einzulassen, und sie verwandelt diese Unverhältnismäßigkeit regelrecht in eine Methode im genausten Hinsehen auf Details, „closest reading“, das blind macht und zugleich blitzartig, ganz nach Benjamin, sichtbare Konfigurationen herstellt, in denen sich die drei Gewaltsysteme ineinander spiegeln, als ob das eine im anderen doch irgendwie erahnt, enthalten, fortgezeugt oder als Antwort und Weiterführung des Vorausgegangenen gewirkt habe. Etwas „Ungerechtes“, auch im juristischen Sinne, entsteht in dieser Arbeitsweise, denn es kann nicht darum gehen, dem einen oder anderen Komplex „gerecht“ zu werden, es kann sich vielmehr „nur“ darum handeln, unverhältnismäßige Lese- und Denkprozesse zu erzeugen, die unsere herkömmlichen Maße durchbrechen. Dafür steht auch die Stimme der Autorin ein, die zuweilen mit ihrem expliziten Ich interveniert, das eine radikale Subjektivität reklamiert, um sich und den Lesenden eine Art Standort zu markieren, von dem aus gesprochen wird und von wo zugleich Bündnisse mit anderen AutorInnen, ForscherInnen und LeserInnen geschlossen werden. Das Experiment dieses außergewöhnlichen Zusammendenkens basiert auf einer langjährigen interdisziplinären, mehrsprachigen, in verschiedenen Ländern erworbenen Wissenschaftskultur der Autorin, verbunden mit gelebter Erfahrung. Es setzt kulturwissenschaftliche mit diskursanalytischen Ansätzen und erinnerungspolitischen Fragen in Beziehung. Anleihen werden darüber hinaus in der Emotionsforschung, der Anthropologie und der Medizingeschichte gesucht, aber auch, bedingt durch Untersuchungen zur Animalisierung von AfrikanerInnen im kolonialen Diskurs, bei den Human-Animal-Studies. Ähnlich vielfältig wie die Methodenzugänge sind die Text- und Bildmaterialien, die von Traumprotokollen über Zeugentexte, literarische Quellen, Forschungsarbeiten und Statistiken, Porträt- und Kunstfotographie bis hin $\mathrm{zu}$ Geschäftsbriefen und Zeichnungen reichen.

3 Ausgangspunkt und roter Faden dieser monumentalen Studie ist das Hörspiel Träume von Günter Eich aus den 1950er Jahren, das Anne D. Peiter mikroskopisch durchforstet und, eine Entdeckung auch für erfahrene Germanistinnen, darin fast alles „erste“ Material für ihre Lesart der drei Gewaltzeiten und -räume findet: Holocaust, Kolonialverbrechen und Hiroshima. Dem Aufspüren dieser Konstellation folgt ein regelrechtes Zudecken mit einer Fülle von Dokumenten, Texten und ikonographischen, vor allem fotographischen Zeugenschaften, die in eine fast unendliche Kette von Korrespondenzen und Widerstreit verwickelt werden. Dabei entsteht zuweilen und überraschenderweise auch eine Form von Verlangsamung, weil in diesem geduldigen Aneinanderreiben immer wieder zu bestimmten Motiven zurückgekehrt wird, die eine Sichtbarkeit durch die Zeit hindurch erleichtern. Ausgehend von der These des Soziologen Erving Goffman, das soziale Leben entspreche der Aufrichtung von Vorderund Hinterbühnen, durch die der Zugang zu bestimmten Realitäten gesellschaftlich reguliert werde, erlegt sich die Autorin und auch uns eine Dynamik der Blicke und des beständigen Blickwechsels auf. Was sieht die Deportierte bei Ruth Klüger, wenn sie durch die Ritzen des Waggons schaut, mit dem sie durch die Landschaft zum Vernichtungslager gefahren wird? Was sieht der deutsche Junge, wenn er auf den Deportationszug schaut? Was sieht die Weimarer Bürgerin, deren Blick aus dem Wohnzimmer auf die Schornsteine des Krematoriums in Buchenwald gerichtet ist? Warum gibt es da ein Gleiten zwischen Sehen und Hinsehen, zwischen Draußen und Drinnen, zwischen Alltag, Normalität und Verbrechen, fragt die Autorin genauestens und analysiert dazu einen Textausschnitt aus Jorge Sempruns Le grand voyage. Von 
diesen Blicken gibt es einen verschlungenen Weg hin auf die quasi unsichtbaren, unerwähnten, bei Eich völlig verstummten Lastenträger im Kolonialismus, ohne welche die Eroberung Afrikas gar nicht möglich gewesen wäre. Hier zeigt die Autorin, wie rassistische Klischees in Verbindung mit Gewaltphantasmen über „die Wilden“ in den Kolonialdiskursen Sklaverei, Verstümmelung und Vergewaltigung der Einwohner auf allen Ebenen zugedeckt und verschwiegen haben. Interessanterweise aber dokumentieren die von Anne D. Peiter zusammen gestellten Fotos, die hier zu sehen auch eines der großen Verdienste der Forscherin ist, im Grunde „alles“: die medizinischen Experimente an den Menschen, die dadurch zu Todkranken werden, die Strafexpeditionen in Form von Amputationen, die Kautschukproduktion, welche die Forscherin wiederum mit dem Motiv des Löwenzahns zusammen bringt, der von der SS im Auschwitzer Nebenlager Rajsko für die Gewinnung von Naturkautschuk eingesetzt werden sollte. Das scheinbar spurenlose europäische Gedächtnis des Kolonialismus sowie des Faschismus reichen sich die Hand, oft bestärkt von literarischen Werken, die ihrerseits die Verdrängung weitertrieben und deren Nachfolgen bis in unserer Jetztzeit hineinragen. So wird gesprungen hin und her, zwischen Landschaften, Objekten, Textsorten, Sprachen und Namen, was nicht jedem Leser gleich leichtfallen wird. Aber mit fortschreitender Lektüre der Habilitationspublikation wird man mit dieser Methodik vertraut, zu der die Autorin uns immer wieder mit Textpassagen des Rückund zugleich Vorausblicks auch Hilfestellungen leistet.

Eine andere Abwesenheit und damit auch epistemologische Problematik zeigt der dritte Teil des Werkes auf, wenn von den fehlenden sinnlichen Erfahrungen mit der Radioaktivität die Rede ist. Die Verfasserin legt hier sehr überzeugend dar, inwiefern sich der Zweite Weltkrieg bei Eich im Kalten Krieg fortschreibt in einem halb offen gelegten Schuld- und Rachediskurs und seinen Allmachts- und globalen Vernichtungsfantasien. Überraschenderweise ist hier die zahlreiche Science-FictionLiteratur, laut Autorin, wenig in der Lage, eine neue écriture zu entwickeln, die etwas erzählen könnte im Hinblick auf das neue Ausmaß der Vernichtung der "ganzen Welt". Anleitung zum Denken findet Anne D. Peiter in diesem Kapitel dafür um so mehr bei Günter Anders, der auf die Entwicklung der "moralischen Vorstellungskraft“ als einzigem Ausweg aus dem, was er als „prometheisches Gefälle“ zwischen dem technisch Machbaren und der Phantasie der historischen Akteure betrachtete, hoffte.

Neben Anders ist auch Karl Kraus eine Art Kompass und Gewährsmann, mit dem sich die Autorin durch ihr großes Werk hindurcharbeitet. Nach dem linguistic turn, wie ihn die Kulturwissenschaften ausgerufen haben, existiert Realität jenseits von Sprache nicht und gilt ein auf Sprache sich gründender Konstruktivismus von "Wirklichkeit“. Sehr feinsinnig fragt Anne D. Peiter in Zusammenhang der von ihr beleuchteten extremsten Gewalterfahrungen im 19. und 20. Jahrhundert immer wieder nach, was ein Sprechen über die Gewalt heute zu leisten hätte, das sich weder mit dem „über“ (das Geschehen und seine Opfer als Objekt) noch mit dem manchmal vorschnellen Unsagbarkeits- und also auch Nichtverstehenstopos zufrieden gibt. Muss hier nicht, wenn es ums „nackte Leben und Überleben“ geht, immer wieder neu und selbstreflexiv nachgedacht werden über das Verhältnis von Sagenmüssen und Konstruktion von Realität durch Sprache, da nichts anderes als genau sie zur Verfügung steht? Und steht aber auch dieselbe Sprache nicht beständig in Gefahr, selbst ideologisch und von Gewalt kontaminiert zu sein und entsprechende Realitäten dadurch herzustellen und weiterzugeben? 
6 Denjenigen, die von den großen Fragen und den winzigen Details umgetrieben werden, die Träume der Gewalt durch zwei Jahrhunderte hindurch uns vorlegt, mit der Aufforderung um lebendige und durchaus kontroverse Verständigung darüber - und ohne Aussicht auf ein Ende dieser immer weiter wirkenden und sich fortzeugenden Verwandtschaften der Gewalt - wird diese Lesezeit als eine sehr besondere im Gedächtnis bleiben. Mit der Fülle der Quellen und Analysen und den vielen losen Enden bietet die Autorin Ausblicke auf zahlreiche Möglichkeiten der weiterführenden Forschung. Ihre Arbeit wird in Zukunft zu einer Referenz werden für Forschende, die sich für das Zusammendenken dieser dreifachen Gewaltgeschichte interessieren. Die Zumutung will uns Mut machen, weiterzudenken. 